\title{
The social dynamics of basins of attraction
}

\author{
$\underline{\text { Sander van der Leeuw }}^{1,2}$ and Carl Folke $^{2,3}$
}

\begin{abstract}
In this paper we conceptualize transformations as societal shifts from one basin of attraction to another. Such shifts occur when a society's information processing system is no longer fit to deal with the dynamics with which the society is involved. To understand when this might be the case, we conceive of a dynamic interaction between two domains, the cognitive one (containing a society's knowledge, values, language, customs, technology etc. that structure information processing) and the environmental one (consisting of the dynamics of the environment within which a society is embedded), which interact through resonance. The two domains are interdependent and coevolve to shape both the information-processing of a society (its culture) and the environment with which it interacts. Crucial in this dynamic is the process of category formation. We used a model that distinguishes between "closed" and "open" categories, which allows us to dynamically relate, but distinguish, a certainty sphere (closed categories dominate), a possibility sphere (open categories dominate), and a problem sphere (absence of categories). Narratives anchor societies' values and dynamics and shape the wider culture of society, making phenomena comprehensible. To foster cultural transitions, narratives need to be modified. To do so, one has to search for narratives in which open categories dominate, and then insert new elements in them. This requires an analysis of the narratives to determine their degree of openness. A tentative approach to such an analysis is offered.
\end{abstract}

\section{INTRODUCTION}

The concepts of "attractor"* and "basin of attraction,"* (see Appendix 1 for definitions of all words with an asterisk) which originated with the emergence of the complex adaptive systems approach (Milnor 1985, Grebogi et al. 1987), are increasingly relevant in efforts to understand how to transform our societies to attain a more sustainable relation between them and the environmental dynamics in which they are embedded (Westley et al. 2011, Carpenter et al. 2019, Schlüter et al. 2019). Yet there is insufficient clarity and agreement on the meaning, function, and impact of these concepts (e.g., https://be-benevolution. com/2020/01/31/attractors/). Here, we present a perspective on the dynamics of societal transformations that details the role of basins of attraction.

In studying the Anthropocene it is not effective to separate societal from environmental dynamics. They are deeply intertwined (Reyers et al. 2018). As expressed by McGlade (1995), there is no social (sub)system nor an environmental one; there are only human perceptions of, and actions on, the integrated, dynamic, social, and natural environment. We therefore adopt for this paper a perspective in which societies and their environments constitute a single, integrated system. But within that system, there are societal dimensions, which humans have the capacity to deal with directly (Brondizio et al. 2016), and environmental dimensions over which the system's influence is more indirect (Steffen et al. 2018).

With the introduction of the Anthropocene as a concept (Crutzen 2002), our societies are no longer seen to be (merely) reacting (adapting) to environmental dynamics, but to be interactive, impacting in a major way on and even shaping the nonhuman dynamics. The scale, connectivity, and speed of the human dimension have become a major driver of Earth system dynamics (Steffen et al. 2018). It follows that in order to understand these integrated dynamics, we need to complement an essentially external, environmental perspective with an internal one, conceiving of the dynamics as societally driven. Thus, as the focus of our research is on the intertwined dynamics of human and nonhuman dimensions, we distinguish, for analytical purposes, within the overall integrated system, an interface between these two dimensions.

Essential to an improved understanding of our current sustainability conundrum, therefore, is knowledge about the interaction between on the one hand our understanding of the realm of external phenomena, and on the other hand our actions upon that realm. Though important efforts have been devoted to studying the dynamics of our environments and their impacts on our societies (e.g., IPCC 2014), comparatively less attention has been devoted to the internal dynamics of our societies and how they impact on and shape our interactions with the environment (Palsson et al. 2013, Brondizio et al. 2016).

Societies selectively define what they consider their environments, what they see as challenges in those environments, and what might be potential solutions for these challenges. Or, as Luhmann (1989:25-26) has phrased it, "societies do not exchange information with the environment, they exchange information about the environment among themselves, in a self-referential manner."

Societies interact with their environment through the filter of their cognition of the phenomena in that environment, i.e., through their information processing apparatus. Only those aspects of the environment that pass through that filter are perceived as socialecological interactions and form the basis for human interpretations, behaviors, norms, and actions relative to the environment. This implies that interactions not captured by a society's information processing remain invisible to society. Such hidden phenomena often appear later as surprises that feed back onto society, generated by broader scale patterns emerging from interactions of local actors and agents (Holling and Meffe 1996, Levin et al. 2013, Schlüter et al. 2019).

${ }^{1}$ Schools of Complex Adaptive Systems (SCAS) and Human Evolution and Social Change (SHESC), Arizona State University, ${ }^{2}$ Beijer Institute of Ecological Economics, Royal Swedish Academy of Sciences, ${ }^{3}$ Stockholm Resilience Centre, Stockholm University 
For its survival, any society has to deal with the environmental conditions it is facing, including those it has created itself because of unintended consequences of its earlier actions. To do so, society's information-processing apparatus must be up to the task. This includes capturing the significant features of the external dynamics and the management and governance of the society's internal dynamics. That apparatus has itself coevolved with the society and its environment, and we need to understand the role of that coevolution in transformations (e.g., Jørgensen et al. 2019).

To apply this to the need for a fundamental organizational transformation of our societies (Westley et al. 2011), we could redefine the current environmental crisis as an incapacity of our societies' information processing to deal with the dynamics in which societies are involved (van der Leeuw 2020a). This perspective highlights the subjectivity of human perceptions of, and actions upon, the environment and emphasizes societies' internal cognitive dynamics. A society's conceptual, institutional, and material organization is seen as part of its information processing apparatus because its ideas, its social organization, and its technology favor certain perceptions and neglect others.

\section{ATTRACTORS AND BASINS OF ATTRACTION}

The focus of this paper is on introducing the concepts of attractor and basins of attraction as tools to investigate the challenge of sustainability, and in particular transformations in the dynamics of social-ecological systems. We borrow these concepts from dynamical systems theory. In the mathematical field of dynamical systems, an attractor* is a set of numerical values toward which a system tends to evolve, for a wide variety of starting conditions of the system. A point attractor is an attractor consisting of a single state. For example, a marble rolling in a smooth, rounded bowl will always come to rest at the lowest point, in the bottom center of the bowl; the final state of motionlessness is a point attractor. A periodic attractor is an attractor consisting of a finite or infinite set of states, where the evolution of the system results in moving cyclically through each state. The ideal orbit of a planet around a star is a periodic attractor, as are periodic oscillations. A strange attractor is an attractor for which the evolution through the set of possible physical states is nonperiodic (chaotic). Most real physical systems (including the actual orbits of planets) involve strange attractors.

It is very common for dynamical systems to have more than one attractor. For each such attractor, its basin of attraction* is the set of points from which the system moves toward that particular attractor. That set of points can be represented as the region of the virtual, dynamic landscape in which the system finds itself and from which the system will necessarily, over time, move toward that attractor. Viewing a basin of attraction as such a region in state space, it has been found that the basic topological structure of such regions can vary greatly from system to system. Thus, the qualitative long-time trajectory of a given system can be fundamentally different depending on which basins of attraction the system interacts with.

For our purposes, we make a distinction between the dynamics of the integrated societal-environmental system and between the dynamics of the societal and the environmental components within that system, as it is the interaction between the latter two that drives the overall system. We represent that interaction as the articulation of the societal component's information-processing apparatus with a series of environmental basins of attraction (Scheffer 2009). That interaction takes the form of a coevolution. Transformations between basins of attraction, occurring at tipping points* may steer the system toward very different future states. If the goal is to redirect the system toward another longterm outcome, as is the case with respect to the sustainability conundrum, the question to ask is therefore, could tweaking basins of attraction and a society's perceptions of them, at least theoretically, influence the trajectory a system takes?

What shapes a society's basin of attraction within the context of the overall integrated system? We argue that it is the ensemble of external (e.g., environmental), internal (societal) and intertwined dynamics that resonate with the cognitive capabilities of a society. One way to represent that is as a dynamic network in which some dynamics may be repelling, while others are attracting. There may also be unperceived dynamics, but society only acts upon the perceived ones. The combination of unperceived and perceived environmental dynamics shapes the basin of attraction. It is important to be aware of the fact that shaping a society's basin of attraction is not a purely self-organizing process in the strict sense, as human information-processing, an inherent part of the overall integrated system, plays a role in shaping the basin, through the feedback loop that constitutes the cognitive filter through which all information is processed.

\section{THE COGNITIVE LOOP}

All perceived interactions between human beings, groups, or societies and their environments (natural as well as societal and technological) pass through the filter of human cognition in the form of information. We propose the following model of that filter, and how it processes information. A loop links the outside world (the realm of phenomena) with the cognitive capacity (the realm of ideas), of the human beings involved (Fig. 1). We conceive of the interaction between the two as a resonance* between a society's internal realm (its information-processing apparatus, including mental and other elements such as language, institutions, and artefacts), and its external realm (the nonhuman environment that society's internal realm interacts with). Observations made about the external realm are interpreted when resonating with existing interpretative schemes in the internal realm, providing information to those schemes.

The patterns that humans derive from their observations selectively reduce the total, almost infinite, complexity of the dynamics observed. When human beings act upon the environment they have observed, their simplified conceptions are confronted with the much more complex dynamics in the outside niche involved. Thus, the resonance triggers challenges to the information-processing apparatus in the form of questions or problems. Meeting these further enhances the processing capacity involved, which will then resonate with new observations, so that the cycle starts anew. As a result, the two domains coevolve to shape both the information-processing capacity of a society and the environment with which it interacts. And because of the difference in dimensionality between the perception and the reality of the environment, any human action upon the latter has unperceived consequences and is subject to "ontological uncertainty" (Lane and Maxfield 1997), the impossibility to predict the outcome of such actions, at least at the systemic level 
and over the longer term. The evolution of a society's cognitive structure drives the trajectory of human-environmental interaction, but only partly directs it.

Fig. 1. The information processing loop (see text; after van der Leeuw 2007).

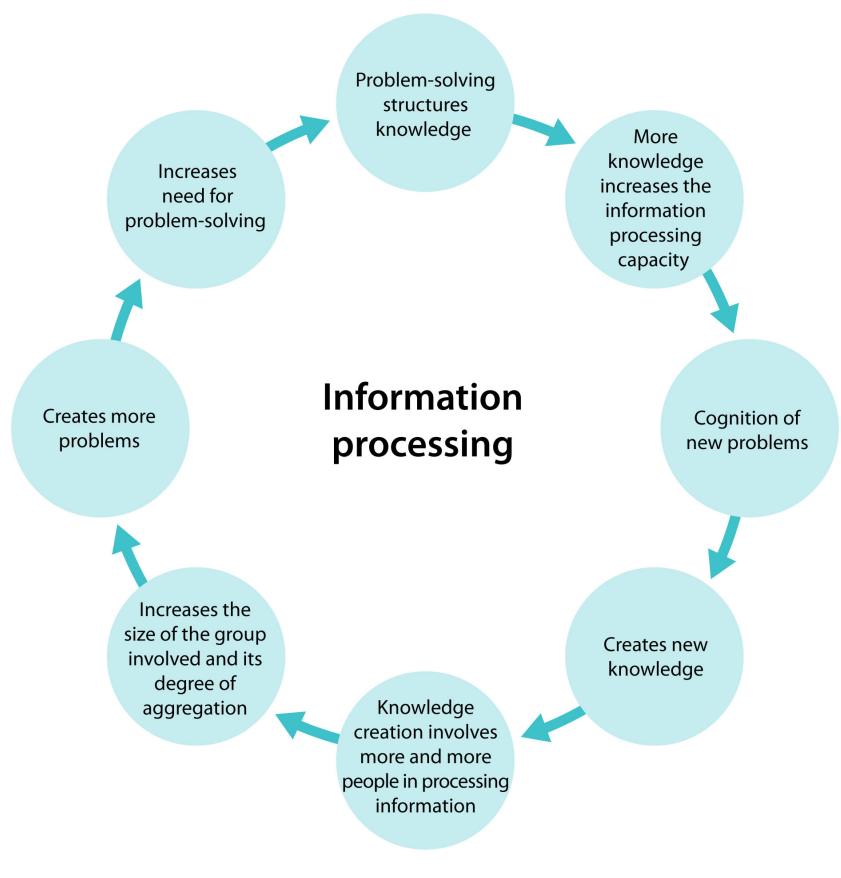

In that trajectory, there will be times when the information processing capacity of the human component of the system resonates with more than one external attractor, for example, when it is on the cusp between two or more basins of attraction. In dynamical systems science, these are the points in a trajectory that may be called "bifurcation points,"* points where the trajectory of the system is faced with two or more potential alternatives. At bifurcation points, the exact dynamics of the resonance are important in determining the trajectory that the system will follow. Coming to understand it for social-ecological systems is currently at the edge of our capability (Scheffer 2009).

The main constraint on the growth of individual or societal information-processing capacity is the development of the human information processing system itself. In the Netherlands, for example, one observes how over six centuries not only the landscape, but also the technology, the spatial organization, the economy, and the administrative institutions emerge from the management of water by the population (van der Leeuw 2012). In the process of expanding human information-processing capacity, some of that capacity is delegated to language, technology, the environment, etc. Concepts and artefacts, for example, fix certain kinds of information processing in the conceptual and linguistic, or the material, technological, or institutional realm by "crystallizing" them as specific "tools for thought and action." They determine that certain actions in the material realm follow set patterns, short-circuiting part of the information-processing involved and alleviating the overall information processing load. In the Dutch example mentioned above, locks and windmills "routinize" aspects of water management so that it no longer takes active information processing to achieve.

In this manner, part of information-processing is simultaneously routinized and displaced outside the mind. The mechanics of a car, for example, relieve the driver from thinking about the engine, reducing the information processing needed for driving to steering, signaling, and regulating the car's speed. At the societal level, the same is ensured by establishing the legal and administrative rules needed to function adequately. In the process, through communication and collective information processing, a society also aligns its members around a set of behavioral rules. These rules enable the members to function collectively and to be selectively aware of their basin(s) of attraction. As the information-processing system evolves, it relates to more and more dimensions of the basin(s) of attraction that shape(s) its trajectory.

\section{INFORMATION PROCESSING, POWER AND INSTITUTIONS}

Each group of human beings thus constructs its information processing apparatus, which anthropologists call "culture" (Wyer 2009). It is formed path-dependently by observing phenomena, interpreting them, testing the interpretation until deemed adequate, then adopting it to observe new phenomena, gain new insights, develop new analytical methods, and further enlarge the scope of the phenomena in a basin of attraction that can be interpreted coherently. The result comprises the ensemble of what the group can collectively perceive and how it cognizes, what it responds to, and how it responds. Culture is thus the result of a long-term path-dependent interaction between the society's information processing and the successive basins of attraction in which it finds itself, in a process that resembles "niche construction" in biology (Odling-Smee et al. 2003). It is an interaction specific for each and every society, group or individual. Although the impact of the society on the basin of attraction that it interacts with reflects that culture, it is only known by the members of the society involved in so far as it resonates with their information-processing apparatus.

Within most societies (except egalitarian ones such as many smallscale hunter-gatherer and early agricultural groups), information processing capacity is unevenly distributed. There are those in society that are placed at the center of the networks that constitute it, and which thus process more information, and more efficiently, than those at the periphery of a society's networks. That difference gives those at the center an advantage over those at the periphery, which manifests itself as a power asymmetry. Such asymmetry has, for example, been demonstrated for the structure of the control network of transnational corporations in relation to global market competition and financial stability, suggesting the existence of an economic super-entity of a small, tightly-knit core of financial institutions (Vitali et al 2011).

People at the center have more dimensions of information processing (knowledge) at their disposal, they are confronted with information earlier, and are thus able to control the information flows through the society by shaping its categories and narratives, as well as the alignment of the other members of society. The alignment of other members is of significance for the emergence 
of institutions ${ }^{[1]}$ as collective information processing tools: behavioral rules in which information processing is regularized with respect to particular subjects or actions.

It has been found that significant differences in collective information processing can be expected among social networks with structural differences in terms of degree of network centralization, density of relations, degree of cohesiveness, and subgroup interconnectivity (Bodin and Crona 2009). Research has also identified the significance of agency in social-ecological network dynamics, like change makers, influencers, leaders, institutional or moral entrepreneurs, or keystone actors in shaping human actions and collaboration (e.g., Westley et al. 2013, Österblom et al. 2017), as reflected in work on sociotechnical transitions, social-ecological transformations, or social innovation (Geels et al. 2017, Westley et al. 2017, HerrfahrdtPähle et al. 2020).

Clearly, power asymmetries impact directly on the institutional framework of a society, for good or for bad, and power dynamics are fundamental in determining the functioning of a society. Those dynamics are partly channeled through the behavioral patterns that are created in any society to underpin its world view, including its power structure and its institutional structure. In shaping those patterns, the categorization of information plays a fundamental role.

\section{THE ROLE OF CATEGORIZATION}

To discuss the dynamics of categorization we will use a simplified model of categorization in relation to decision making under uncertainty (Tversky 1977, Tversky and Gati 1978, Kahnemann et al. 1982; Fig. 2). ${ }^{[2]}$ Categorization combines a number of phenomena into a category that is distinguishable by adopting one or more of their characteristics as a label. That process requires pattern recognition, a comparison between similarities and dissimilarities among the phenomena observed. In the first phase of that process, the category is the subject and the phenomena is the referent. There is thus a bias in favor of similarity: an emphasis on what might unite the observed phenomena into a category. But when the relevant category is firmly established, the process is inverted: the categories become the referents and the phenomena the subjects, so that the comparisons are biased toward dissimilarity, and it is determined which phenomena, after all, did not belong in the categories established.

Fig. 2. Category formation according to Tverski and Gati 1978 (illustration from van der Leeuw 2020a).

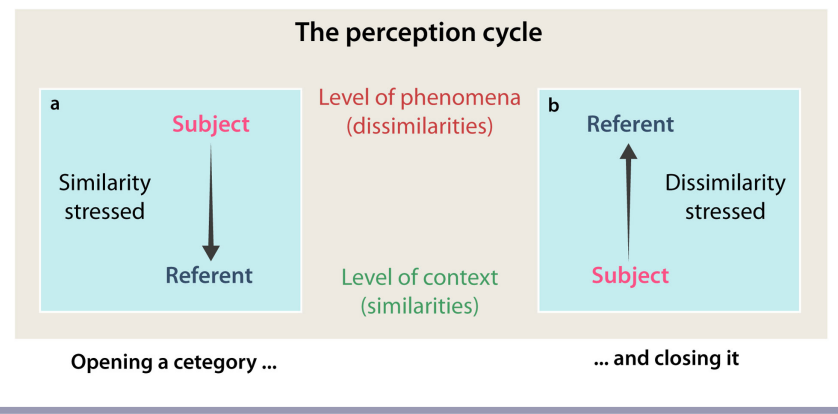

We all know this phenomenon in our scientific work since Kuhn's research on scientific revolutions (1962). When faced with unknown phenomena, we first create open categories by developing hypotheses about those phenomena that emphasize their similarity. Little by little, we then whittle away at the phenomena concerned by the hypotheses, in order to get a better handle on them. In so doing, we emphasize what seem the most important dimensions of the phenomena concerned, thus transforming the hypotheses into definitions (closed categories). And once we have defined a set of categories, those codetermine further investigations on different phenomena, so that a pathdependent* knowledge structure is built up.

We can also describe this process as follows (Rescher 1964). First, we consider an extrinsically defined group, which is circumscribed in space and time, and therefore does not include all the potential other entities that may ultimately be found to belong in the category. That category is therefore essentially open. Once we have constructed a coherent framework to include both the relevant members of the group and other potential members (outside the group) considered similar, we transform the group into an (intrinsically defined) class on the basis of characteristics that apply to all actual and prospective members. In the first part of this process, the reasoning is inductive, from particular phenomena to more general theories, while in the second part it is deductive, from the theories to the particular phenomena to which they apply (van der Leeuw 1976).

As a result of this process, in the virtual cognitive space of a society one can distinguish three different "cognitive spheres" into which one might divide the overall information processing apparatus in the minds of people and groups (van der Leeuw 1993):

- A "certainty sphere," which is made up of the "closed" categories, ensuring that a person, group, or society knows exactly what is what, and has fixed ideas on how to proceed;

- A "possibility sphere," which consists of the "open" categories, about which the group is still to some extent undecided, and therefore flexible in its interactions;

- A "problem sphere," consisting of the domain for which there are no categories (yet), and which therefore is that of unknown or dimly perceived, unsolved challenges.

Thus, in the interaction between the information processing and the basin of attraction in which an individual or a society finds itself, the "mind" distinguishes three kinds of phenomena: known unknown ones, tentatively known (hypothetical) ones, and definitely known ones. The mix between these will determine the way in which the societal component of the system interacts with the basin of attraction in which it finds itself.

If we then consider in detail how the problem sphere comes into being, one needs to take into account that human perception of the present iteratively relates information about an assumed past to information about personal experience in the present, and projects the resulting vector into the future. In other words, there is an interaction between perception from an a posteriori perspective (on the past) which limits variation, and perception from an a priori point of view (on the future), which opens opportunity for variation. The latter is focused on emergence, on novelty, and on possibilities and probabilities ("opening" 
categories), while the former is focused on origins, on tradition, and on causality ("closing" categories). It is in that interaction that invention takes place. Often, crises (perceived or real) open up space for an intense interplay between such memory and novelty that may lead to shifts in basins of attraction (Gunderson and Holling 2002). This delicate interplay has been demonstrated in case studies of governance transformations from local to global levels, for example, in the management of a semiurban landscape in Sweden, or coastal fisheries in Chile, to stewardship of the Great Barrier Reef or global adaptive governance of fisheries in the Southern Ocean (Olsson et al. 2004, 2008, Gelcich et al. 2010, Österblom and Folke 2013, Schultz et al. 2015).

\section{THE ROLE OF NARRATIVES}

What anchors a culture is an incredibly complex dynamic between a society's cognitive dynamics, its network structures, and its environmental context, all interacting within the path-dependent trajectory that shaped its dynamics. As a function of these a society defines its identity, shapes the epistemology through which it interacts with its environment, establishes its fundamental values, and much more. All these are deeply anchored in the minds of its members, and determine their outlook, their opinions, and their decisions. This anchor is so complex that we can only partly hope to unravel it, map it, understand it, or modify it.

The way to approach that problem is through the identification and modification of the culture's narratives* (van der Leeuw 2020b). Narratives occur in all societies, from the distant past (e.g., the third millennium BCE Gilgamesh epic in Mesopotamia) to the present and have a similar function: as superficially linear (and therefore low-dimensional) told or written stories, they summarize events in a highly multidimensional world by referring to "Gestalts."* Such narratives emerge to explain an unknown phenomenon in terms of the culture that experiences it. The narrative acculturates a phenomenon into the wider culture of the society involved.

The "Gestalts" involved are multidimensional and deeply anchored in the culture, so that the narratives, or myths, connect the people among whom they are told with their culture. A narrative asserts their identity and their way of organizing themselves and the world around them, and thus also their ways of doing things. The link between the (uni-)linear stories and the multidimensional world is constructed through resonance: the narrative refers to multidimensional characters that are part of the symbolic riches of the cultures involved.

\section{IMAGINED FUTURES}

Jens Beckert (2016) argues that in Western societies, human decisions and actions are driven by "imagined futures." Since 1750, according to him, as the Western perspective on the future opened up (Girard 1990), this set in motion a (uniquely Western) cognitive feed-forward loop that creates in our individual and collective minds "imagined futures" and then develops "fictional expectations" that motivate people toward realizing them. In Beckert's words: "... expectations of the unforeseeable future inhabit the mind not as foreknowledge but as contingent imaginaries"(2016:9) ... "they create a world of their own into which actors can (and do) project themselves" (2016:10). These fictional expectations are anchored in narratives that are continually adapted.
The exchange between such imagined futures and present conditions shapes the narratives people develop or adhere to, which in turn drive our imagined futures and our decision making. Hence, "fictionality, far from being a lamentable but inconsequential moment of the future's fundamental uncertainty, is a constitutive element of capitalist dynamics, including economic crises" (Beckert 2016:12). He illustrates that in his book in detail for four main pillars of any economy: money, credit, investment, and innovation.

The implications for the role of narratives in shaping our imagined futures stretch far beyond the economy. First, narratives express the cultural, institutional, and social embeddedness of our human decision making. Decisions reflect the value systems of the people concerned; they are shaped in the interaction networks of these people. The UN's Sustainable Development Goals, for example, are in essence based on a Western imagined future of continued "progress" that, as part of globalization, has been projected onto other cultures. In other parts of the world, one finds very different imagined futures underneath that global projection.

Second, individual and shared anticipations of the future are constructed by comparing the present to an experienced past and an imagined future (van der Leeuw 2020b), and they are maintained only as long as there is confidence in that future, and by implication as long as the balance between open and closed categories is in favor of the latter. In the absence of such confidence, when open categories dominate, a degradation in the clarity of a society's perceptions and certainties, or even a crisis, is experienced.

The anticipatory loop can then, very rapidly, be turned in a negative direction characterized by self-fulfilling negative dynamics driving toward increased uncertainty, as in the case of the recent financial crisis. But this is not confined to such sharp crises; it can also slowly undermine the totality of our confidence in the future and result in hesitations, contradictory actions, and general loss of self-confidence.

Third, we need to consider the relationship between our imagined futures and the "real world out there." That interaction is clearly an open-ended one that is not fully controllable. As anticipated futures are confronted with the material and social "real" world, it is impossible to predict the outcome of such confrontations, especially over the longer term, because of changes in the secondorder dynamics of the context in which shorter term decisions are made. This can theoretically very rapidly transform peace into war, progress into the opposite, trust into distrust.

But shocks, extreme events or real world crises may also unlock competing narratives and imagined futures that have slowly emerged and been in the shadow, waiting in the wings for windows of opportunities to transition communities, economies, societies, onto other pathways (Gunderson et al. 1995, Gelcich et al. 2010, Herrfahrdt-Pähle et al. 2020). Such events may also move the information processing loop (Fig. 1) into systemic understanding of the bigger picture, triggering paradigm shifts, spreading social innovation, and new forms of collective action (Olsson et al. 2008, Westley et al. 2017, Carpenter et al. 2019). 


\section{A SOCIETY ALWAYS SITS ASTRIDE DIFFERENT FIELDS OF TENSION AND HAS TO MAKE CHOICES}

Could tweaking basins of attraction and a society's perceptions of them, at least theoretically, influence the trajectory a system takes? To address that question, we must first look at the wider dynamic landscape around a society. It encompasses numerous basins of attraction. A society's trajectory is therefore in part determined by the fields of tension existing between these basins of attraction. As the system's trajectory unfolds, there will be moments when those tensions make themselves felt or seen, so that the need for systemic choices between different trajectories becomes clear. In complex systems language, one speaks of "bifurcation points," points in the trajectory at which it is necessary for human information processing to adopt a radically different structure ("paradigm change") to enable a society to continue to coherently exist. One such moment in our history is the present one, at which a transformation in our fundamental societal values and culture is called for if we, as a human species, are to find a sustainable relationship with the broader environment, the Earth system and its biosphere, which we are embedded within and dependent upon (Folke et al. 2016).

That raises two further questions: Can we influence the way in which a societal system proceeds once it has reached a bifurcation point? If we can, how do we do so? We will devote much of the remainder of this paper to these two questions. The core of both questions lies in the relationship between the domain of ideas and that of phenomena, between the information processing apparatus available to the society and the environment in which it is embedded, and with which it interacts. If we were to be able to influence the former, that would imply that other aspects of the latter were cognized, and that the realm of phenomena would resonate differently with the cognitive realm. This is what underlies efforts toward sustainable futures or biosphere stewardship (Westley et al. 2011, Bennett et al. 2016, Folke et al. 2016). In effect, the basin of attraction would be modified and the society's trajectory would become subject to the influence of other attractors. So, the first question is: What anchors a society's basin of attraction?

\section{WHAT ANCHORS A BASIN OF ATTRACTION?}

In a study of the dynamics of innovation in technology, one of us came to conclude that any approach to exercising a technique is anchored at minimally three different levels, in increasing order of flexibility and opportunity for change (van der Leeuw 1993):

1. The slowest to change is the collective knowledge that is shared between the members of the community involved. Change at this level involves changing the worldview of the community, its habitus, its approach to technology and the world in general. The main barrier to such change is that the perspective of the community is limited by the things it has never thought about and which it therefore has no way to describe, analyze, or conceptualize. Breaking through that barrier is itself a major invention/innovation.

2. At the level of the individual one has to take tacit knowledge (know-how) into account, which has either been subsumed under more conscious conceptual knowledge and customs or resides in the physical, neuro-muscular behavior of the human body. It is difficult to change because it is not embedded in our conscious memory but is exercised as routine, without conscious thought.
3. But the individual also has conscious knowledge (know that), which is subject to conscious learning and is therefore the easiest and quickest to change. It actively involves the conscious mind, planning and changing behavior. Yet one must remember that such conscious knowledge is also limited by its boundary with the unknown: those processes, questions, and challenges that one has never thought about. It is in this domain that changes are made most easily.

As we have argued above, categorization is fundamental to shaping human perception of the world. It is reflected in the narratives that maintain a particular outlook. The three levels just mentioned distinguish between the narratives (or parts thereof) that are open to change, and those that are not. If the narrative is predominantly constituted of closed categories, it will fall in category 1 , the collective knowledge that is difficult to change. But if open categories dominate, there is a degree of indeterminacy about the narrative that implies that variations can be considered in the implementation of the narrative concerned, as in the case of category 2. Category 3 refers to the resources involved in the implementation of the narratives; these are easiest to change. Knowing this, the next question is of course the following: Where and how does change occur?

\section{THE DYNAMICS OF CHANGE AS ARTICULATION BETWEEN THE COGNITIVE AND THE ENVIRONMENTAL DOMAINS}

The challenge, if one wants to change narratives is therefore twofold. First, one has to identify narratives that are changeable because they include a number of open categories, and then one has to insert elements in those narratives that favor the system to change direction. To illustrate this, we focus on how such a move could occur in a technology. ${ }^{[3]}$

Much of the current advertising industry depends on designing narratives that change people's attitudes to particular products or ways of doing things. A beautiful published example of the challenges encountered in changing people's attitudes, but also the success achieved when this is done, is the story of Lane and Maxfield (2009) on the introduction of distributed control networks (LonWorks) by Echelon, against the established vision of the control industry (Honeywell et al.), which saw control always centralized. An important element in this story is the role of the general cultural background in the acceptance of such narratives. In this case, whereas development of the technology initially was lauded but then hindered in the U.S., it was accepted in Italy.

At the interface between society and the environment, technologies do not follow either the logic of the society or that of an external basin of attraction, but are determined by the resonance between them. The constraints and affordances of the material world constitute the external niche, and the perspective of the person shaping the technology constitutes the internal niche. Because of the limitations of our short-term working memory*, the latter is always a simplified and biased version of the former (Read and van der Leeuw 2008). Though change may be either exogenous (e.g., a change in raw material use because of an environmental change) or endogenous (e.g., a change in perception that introduces a change in the manufacturing process, thus requiring a different raw material), in both cases it is the change in the internal niche, the information processing by the maker, that instantiates the technology change. 
Narratives reflect the state of the society's information processing apparatus. Thus, a change in narrative is always the driver as well as the signal of a transition between basins of attraction, whether driven by exogenous or by endogenous dynamics, or a combination of both. Such changes in narrative occur regularly in any interaction between the cognitive apparatus of a society and the dynamics of its environment, including consecutive basins of attraction. As mentioned, in science we call them changes of paradigm, occurring when more and more phenomena cannot be explained by the pre-existing one (Kuhn 1962).

Over time, such adjustments between a society's information processing and the succession of basins of attraction that impact on it, engender a coevolution that in turn shapes the wider context of further change. Innovations create a new dependency relationship with both societies' information-processing apparatus and their environmental context (basin of attraction), and when that context changes, the invention may well disappear or be transformed. Conversely, if the innovation is no longer produced, it will disappear. In such a technological coevolution, each and every choice, once made, limits the total option set for future choices and generates its own set of unintended consequences, eventually leading to new innovations. The domain in which material and procedural inventions occur, which we could call the "technosphere"* (Haff 2014), generates its own dynamics, which does in part shape, but is also shaped by, the path dependency of a society around its evolving technology.

The same is true of every social, organizational, and institutional choice made. We can extend this model of technological change to the wider area of society's nontechnological narratives, concerning societal and cultural changes. On the one hand, the model of the external environment should be as complete and unbiased as possible, referring to the different relevant functions, materials, techniques, institutions, etc. that constitute that context in the world out there. On the other, the internal niche should include the actor's subjective views, as reflected in the relevant narratives relating to that context. That latter perspective is always partial, biased, and part-driven by social, cultural, personal, and other factors external to the context of innovation, and its object of study is how the maker's perception articulates these factors with the external world (van der Leeuw 2020a).

\section{ATTRACTORS AND BASINS OF ATTRACTION FOR SUSTAINABILITY}

We have suggested that narratives serve as social attractors toward which behavior, society, and culture self-organize. We have also proposed that guided by an overall narrative(s), the complex selforganization dynamics between the collective mind of a society with its information-processing tools and the dynamics of a society's environment - the adaptive dance of the cognitive and the environmental domains - coevolves and shapes basins of attraction. As a result, some of these basins remain shallow and easy to change, while others become deep, persistent, and difficult to move out of.

All human groups operate around shared narratives, which create identity, meaning, and core values and shape the epistemology through which they interact with the broader environment. All these are deeply anchored in the minds of its members, and frame their outlook, their opinions, and their decisions, consciously or unconsciously. Some examples of such more or less persistent systemic narratives that have shaped society, culture, and civilization are Christianity in Western Europe, Communism in the USSR and Eastern Europe, Manifest Destiny in the USA, indigenous cosmologies among the original inhabitants of the Americas, Sunni and Shiite Islam in many parts of Asia, Fascism in 20th century Western European and South American countries. Of course, these are examples at the most general level for large numbers of people. Narratives adhered to by smaller subsections of such populations are often embedded in these larger narratives, such as Protestant, Catholic and Orthodox narratives among the Christian populations of Europe, or, at an even lower level, the narratives of the various evangelical sects in North America.

As we have proposed, an observed change in narratives is a central driver as well as a signal of the likelihood of a transition between basins of attraction, whether driven by exogenous or by endogenous dynamics, or a combination thereof. When open categories dominate in a narrative, that narrative is to some extent open to change, but when closed categories dominate, it is not.

We have also proposed that changes in narrative occur when there is an increasing mismatch of the interaction between the cognitive apparatus of a society and the dynamics of its environment, that is, when more and more phenomena cannot be explained by the pre-existing narrative. This is the time for paradigm shifts, or shifts in basins of attraction, and the emergence of new basins of attraction.

There is a discussion whether or not contemporary globalized society is approaching such a shift (or alternatively, that many contemporary societies and cultures are at the brink of such shifts), whether or not open or closed categories dominate the narrative. In science, this is reflected in the growing number of calls for transformative change toward sustainability (Westley et al. 2011, Hackmann and St. Clair 2012). In society, social discontent and rising fluctuations characterize a phase of uncertainty and exploration (Carpenter et al. 2019), and extreme events and shocks have exposed the tightly connected globalized world and its associated vulnerabilities (Nyström et al. 2019), exposing, for example, the vulnerability of the globally interconnected food system (Cottrell et al. 2019).

How can we distinguish closed from open categories in narratives? A promising way forward, tested as part of the ARCHAEOMEDES project undertaken in the 1990s, is a method developed for analyzing interviews (B. Wirtz and R. Langevin 1997, unpublished manuscript). By scanning through each interview from beginning to end, the authors looked at the full interview sequence as a whole, and calculated the expected and observed entropy* of the individual statements in the interview. They thereby retained the information conveyed by the internal chronology and the contextual dynamics of the interviews, and were able to compare the mode of expression of the speaker on different topics. The difference between expected and observed entropy varied within interviews, along with the topics the interviews were talking about. If the interviewees were talking about the past, for example, life as they lived it in their youth, there would be much less difference between the two entropies than if they were talking about more recent, personal experiences. In that case, the observed entropy was much more important than the expected one. This reflects that less certainty existed about these recent experiences than about "the way things were in the 
past." In other words, the narrative was dominated by closed categories when discussing the past, as if those discussions were anchored in a canonical vision of that past, whereas in discussing personal experiences in the recent past and the present there was a much higher observed entropy, and thus less certainty about the narrative.

Confrontation with uncertainty requires capacities to be able to live with changing circumstances, to keep options alive. Such "social resilience" refers to the capacity of individuals, groups, communities, societies, and civilizations to secure acceptable outcomes (material, symbolic, emotional) under new circumstances and when necessary by new means, even when this entails significant modifications to behavior or to the social frameworks, the narratives that structure and give meaning to behavior (Hall and Lamont 2013).

Narratives of hope for transformations toward sustainable futures are in demand. They should broaden cultural membership by promoting new narratives that resonate, inspire, and provide hope centered on a plurality of criteria of worth and social inclusion (Lamont 2019). Narratives of hope as new attractors for shifting basins of attraction toward sustainability (Otto et al. 2020) represent ideas about "imagined futures" or alternative ways of visualizing and conceptualizing what has yet to happen and motivate action toward new development pathways (Milkoreit 2017). As they circulate and become more widely shared, such imagined futures have the potential to foster predictable behaviors. These in turn can stimulate new laws, regulations, and investments in research and development of new (social and material) technologies that fit the aspirations of the imagined futures (Beckert 2016).

We sense that a collective narrative may be on the rise with new imagined futures of hope emerging, and increasingly dominated by open categories. Such new attractors, currently being formed, seem to appreciate humanities' interdependence with the planet we are living on, the fact that all societies are embedded in the biosphere and what this means for the well-being and even survival of civilizations. This new "collective mind" of society is coevolving with the dynamics of the realities of the Anthropocene. It will hopefully foster capacities for redirecting societal development into basins of attraction ensuring sustainable futures, with social, economic, and political systems acting on such a narrative.

\section{CONCLUSION}

We have proposed a model to describe and analyze the dynamics of interaction between a societal system and the basins of attraction surrounding it. The basis of this model is the assumption that there are two domains that shape and construct each other in mutual interaction, one serving as the societal information-processing apparatus (the collective mind and its information-processing tools), and the other encompassing the dynamics of the society's environment.

As a societal system follows its trajectory, it will inevitably come to bifurcation points where it experiences a transition from one basin of attraction to the next. We have therefore also described very briefly what happens at such a point of transition.

All this raises the question how the (collective) societal information processing apparatus functions. We have proposed a model of the dynamics of categorization, and its role in shaping the narratives that ensure the coherence of the society's values and culture. When open categories dominate in a narrative, that narrative is to some extent open to change, but when closed categories dominate, it is not. Hence, as discussed in the last section, the core issue from the perspective of attempting to move a societal system into a different trajectory and basin of attraction, is whether we can identify open and closed parts of narratives, and then modify the open ones in a direction that moves our societies closer to sustainability.

${ }^{[1]}$ The concept of institution is here used in the anthropological sense, comprising collective ways of thinking about, and doing things, which are not necessarily formalized as they are in current day societies.

${ }^{[2]}$ For a more extensive summary, see van der Leeuw $2020 a$.

${ }^{[3]}$ For a more extensive explanation of this part of our model, see van der Leeuw $2020 a$.

Responses to this article can be read online at: https://www.ecologyandsociety.org/issues/responses. php/12289

\section{Data Availability:}

Datalcode sharing is not applicable to this article because no new datalcode were created or analyzed in this study.

\section{LITERATURE CITED}

Beckert, J. 2016, Imagined futures: fictional expectations and capitalist dynamics. Harvard University Press, Cambridge, Massachusetts, USA. https://doi.org/10.4159/9780674545878

Bennett, E. M., M. Solan, R. Biggs, T. McPhearson, A. V. Norström, P. Olsson, L. Pereira, G. D. Peterson, C. RaudseppHearne, F. Biermann, et al. 2016. Bright spots: seeds of a good Anthropocene. Frontiers in Ecology and the Environment 14:441-448. https://doi.org/10.1002/fee.1309

Bodin, Ö., and B. I. Crona. 2009. The role of social networks in natural resource governance: what relational patterns make a difference? Global Environmental Change 19:366-374. https://doi. org/10.1016/j.gloenvcha.2009.05.002

Brondizio, E. S., K. O’Brien, X. Bai, F. Biermann, W. Steffen, F. Berkhout, C. Cudennec, M. C. Lemos, A. Wolfe, J. PalmaOliveira, and C.-T. A. Chen. 2016. Re-conceptuaizng the Anthropocene: a call for collaboration. Global Environmental Change 39:318-327. https://doi.org/10.1016/j.gloenvcha.2016.02.006

Carpenter, S. R., C. Folke, M. Scheffer, and F. R. Westley. 2019. Dancing on the volcano: social exploration in times of discontent. Ecology and Society 24(1):23. https://doi.org/10.5751/ES-10839-240123

Cottrell, R. S., K. L. Nash, B. S. Halpern, T. A. Remeny, S. P. Corney, A. Fleming, E. A. Fulton, S. Hornborg, A. Johne, R. A. Watson, and J. L. Blanchard. 2019. Food production shocks 
across land and sea. Nature Sustainability 2:130-137. https://doi. org/10.1038/s41893-018-0210-1

Crutzen, P. J. 2002. Geology of mankind. Nature 415:23. https:// doi.org/10.1038/415023a

Folke, C., R. Biggs, A. V. Norström, B. Reyers, and J. Rockström. 2016. Social-ecological resilience and biosphere-based sustainability science. Ecology and Society 21(3):41. https://doi.org/10.5751/ ES-08748-210341

Geels, F. W., B. K. Sovacool, T. Schwanen, and S. Sorrell. 2017. Sociotechnical transitions for deep decarbonization. Science 357:1242-1244. https://doi.org/10.1126/science.aao3760

Gelcich, S., T. P. Hughes, P. Olsson, C. Folke, O. Defeo, M. Fernández, S. Foale, L. H. Gunderson, C. Rodríguez-Sieker, M. Scheffer, R. S. Steneck, and J. C. Castilla. 2010. Navigating transformations in governance of Chilean marine coastal resources. Proceedings of the National Academy of Sciences of the USA 107:16794-16799. https://doi.org/10.1073/pnas.1012021107

Girard, R. 1990. Innovation and repetition. SubStance 19 (62/63):7-20. https://doi.org/10.2307/3684663

Grebogi, C., E. Ott, and J. A. Yorke. 1987. Chaos, strange attractors, and fractal basin boundaries in nonlinear dynamics. Science 238(4827):632-638. https://doi.org/10.1126/science.238.4827.632

Gunderson, L. H., and C. S. Holling, editors. 2002. Panarchy: understanding transformations in human and natural systems. Island, Washington, D.C., USA.

Gunderson, L. H., C. S. Holling, and S. Light, editors. 1995. Barriers and bridges to the renewal of ecosystems and institutions. Columbia University Press, New York, New York, USA.

Hackmann, H., and A. L. St. Clair. 2012. Transformative cornerstones of social science research for global change. Report of the International Social Science Council, Paris, France. [online] URL: https://www.belmontforum.org/wp-content/uploads/2012/06/ issc transformative cornerstones report.pdf

Haff, P. 2014. Humans and technology in the Anthropocene: six rules. Anthropocene Review 1(2):126-136. https://doi. org/10.1177/2053019614530575

Hall, P. A., and M. Lamont, editors. 2013. Social resilience in the neoliberal era. Cambridge University Press, Cambridge, UK.

Herrfahrdt-Pähle, E., M. Schlüter, P. Olsson, C. Folke, S. Gelcich, and C. Pahl-Wostl. 2020. Sustainability transformations: sociopolitical shocks as opportunities for governance transitions. Global Environmental Change 63:102097. https://doi.org/10.1016/ j.gloenvcha.2020.102097

Holling, C. S., and G. K. Meffe. 1996. Command and control and the pathology of natural resource management. Conservation Biology 10:328-337 https://doi.org/10.1046/j.1523-1739.1996.10020328. $\underline{\mathrm{X}}$

Intergovernmental Panel on Climate Change (IPCC). 2014. Climate change 2014: Synthesis report. Contribution of Working Groups I, II and III to the Fifth Assessment Report of the Intergovernmental Panel on Climate Change. Core Writing Team, R. K. Pachauri, and L. A. Meyer, editors. IPCC, Geneva, Switzerland.
Jørgensen, P. S., C. Folke, and S. P. Carroll. 2019. Evolution in the Anthropocene: informing governance and policy. Annual Review of Ecology, Evolution, and Systematics 50:527-546. https:// doi.org/10.1146/annurev-ecolsys-110218-024621

Kahnemann, D., P. Slovic, and A. Tversky, editors. 1982. Judgment under uncertainty: heuristics and biases. Cambridge University Press, Cambridge, UK. https://doi.org/10.1017/ CBO9780511809477

Kuhn, T. S. 1962. The structure of scientific revolutions. University of Chicago Press, Chicago, Illinois, USA.

Lamont, M. 2019. From 'having' to 'being': self-worth and the current crisis of American society. British Journal of Sociology 70:660-707. https://doi.org/10.1111/1468-4446.12667

Lane, D. A., and R. Maxfield. 1997. Foresight, complexity and strategy. Pages 169-198 in W. B. Arthur, S. N. Durlauf, D. A. Lane, editors. The economics as an evolving complex system II. Westview, New York, New York, USA. https://doi.org/10.1201/9780429496639-7

Lane, D. A., and R. R. Maxfield. 2009. Building a new market system: effective action, redirection and generative relationships. Pages 263-288 in D. Lane, D. Pumain, S. E. van der Leeuw, and $\mathrm{G}$. West, editors. Complexity perspectives in innovation and social change. Methodos Series: Methodological Prospects in the Social Sciences, Vol 7. Springer, Dordrecht, The Netherlands. https:// doi.org/10.1007/978-1-4020-9663-1 10

Levin, S. A, T. Xepapadeas, A.-S. Crépin, J. Norberg, A. de Zeeuw, C. Folke, T. P. Hughes, K. Arrow, S. Barrett, G. Daily, P. Ehrlich, N. Kautsky, K.-G. Mäler, S. Polasky, M. Troell, J. Vincent, and B. H. Walker. 2013. Social-ecological systems as complex adaptive systems: modeling and policy implications. Environment and Development Economics 18:111-132. http://dx.doi.org/10.1017/ $\underline{\mathrm{S} 1355770 \mathrm{X} 12000460}$

Luhmann, N. 1989. Ecological communication. University of Chicago Press, Chicago, Illinois, USA.

McGlade, J. 1995. Archaeology and the ecodynamics of humanmodified landscapes. Antiquity 69(262):113-132. https://doi. org/10.1017/S0003598X00064346

Milkoreit, M. 2017. Imaginary politics: climate change and making the future. Elementa: Science of the Anthropocene 5:62. https://doi.org/10.1525/elementa.249

Milnor, J. 1985. On the concept of attractor. Communications in Mathematical Physics 99:177-195. https://doi.org/10.1007/ BF01212280

Nyström, M., J.-B. Jouffray, A. B. Norström, B. Crona, P. S. Jørgensen, S. R. Carpenter, Ö. Bodin, V. Galaz, and C. Folke. 2019. Anatomy and resilience of the global production ecosystem. Nature 575:98-108. https://doi.org/10.1038/s41586-019-1712-3

Odling-Smee, F. J., K. N. Laland, and M. W. Friedman. 2003. Niche construction: the neglected process in evolution. Princeton University Press, Princeton, New Jersey, USA.

Olsson, P., C. Folke, and T. Hahn. 2004. Social-ecological transformation for ecosystem management: the development of adaptive co-management of a wetland landscape in southern Sweden. Ecology and Society 9(4):2. https://doi.org/10.5751/ ES-00683-090402 
Olsson, P., C. Folke, and T. P. Hughes. 2008. Navigating the transition to ecosystem-based management of the Great Barrier Reef, Australia. Proceedings of the National Academy of Sciences of the USA 105:9489-9494. https://doi.org/10.1073/pnas.0706905105

Österblom, H., and C. Folke. 2013. Emergence of global adaptive governance for stewardship of regional marine resources. Ecology and Society 18(2):4. http://dx.doi.org/10.5751/ES-05373-180204

Österblom, H., J.-B. Jouffray, C. Folke, and J. Rockström. 2017. Emergence of a global science-business initiative for ocean stewardship. Proceedings of the National Academy of Sciences of the USA 114:9038-9043. https://doi.org/10.1073/pnas.1704453114

Otto, I. M., J. F. Donges, R. Cremades, A. Bhowmik, R. J. Hewitt, W. Lucht, J. Rockström, F. Allerberger, M. McCaffrey, S. S. P. Doe, A. Lenferna, N. Morán, D. P. van Vuuren, and H. J. Schellnhuber. 2020. Social tipping dynamics for stabilizing Earth's climate by 2050. Proceedings of the National Academy of Sciences of the USA 117:2354-2365. https://doi.org/10.1073/ pnas. 1900577117

Palsson, G., B. Szerszynski, S. Sörlin, J. Marks, B. Avril, C. Crumley, H. Hackmann, P. Holm, J. Ingram, A. Kirman, M. Pardo Buendía, and R. Weehuizen. 2013. Reconceptualizing the 'Anthropos' in the Anthropocene: integrating the social sciences and humanities in global environmental change research. Environmental Science and Policy 28:3-13. http://dx.doi. org/10.1016/j.envsci.2012.11.004

Read, D. W., and S. van der Leeuw. 2008. Biology is only part of the story ... Philosophical Transactions of the Royal Society, Series B 363:1959-1968. https://doi.org/10.1098/rstb.2008.0002

Rescher, N. 1964. Introduction to logic. St Martin's Press, New York, New York, USA.

Reyers, B., C. Folke, M.-L. Moore, R. Biggs, and V. Galaz. 2018. Social-ecological systems insights for navigating the dynamics of the Anthropocene. Annual Review of Environment and Resources 43:267-289. https://doi.org/10.1146/annurev-environ-110615-085349

Scheffer, M. 2009. Critical transitions in nature and society. Princeton University Press, Princeton, New Jersey, USA.

Schlüter, M., L. J. Haider, S. J. Lade, E. Lindkvist, R. Martin, K. Orach, N. Wijermans, and C. Folke. 2019. Capturing emergent phenomena in social-ecological systems: an analytical framework. Ecology and Society 24(3):11. https://doi. org/10.5751/ES-11012-240311

Schultz, L., C. Folke, H. Österblom, and P. Olsson. 2015. Adaptive governance, ecosystem management, and natural capital. Proceedings of the National Academy of Sciences of the USA 112:7369-7374. https://doi.org/10.1073/pnas. 1406493112

Steffen, W., J. Rockström, K. Richardson, T. M. Lenton, C. Folke, D. Liverman, C. P. Summerhayes, A. D. Barnosky, S. E. Cornell, M. Crucifix, J. F. Donges, I. Fetzer, S. J. Lade, M. Scheffer, R. Winkelmann, and H. J. Schellnhuber. 2018. Trajectories of the Earth system in the Anthropocene. Proceedings of the National Academy of Sciences of the USA 115:8252-8259. https://doi. org/10.1073/pnas.1810141115
Tversky, A. 1977. Features of similarity. Psychological Review 84:327-352. https://doi.org/10.1037/0033-295X.84.4.327

Tversky, A., and I. Gati. 1978. Structures of similarity. Pages 79-98 in E. Rosch and B. B. Lloyd, editors. Cognition and categorization. Lawrence Erlbaum, Hillsdale, New Jersey, USA.

van der Leeuw, S. E. 1976. Studies in the technology of ancient pottery. Two volumes. Dissertation. Amsterdam University Press, Amsterdam, The Netherlands.

van der Leeuw, S. E. 1993. Giving the potter a choice: conceptual aspects of pottery techniques. Pages 238-288 in P. Lemonnier, editor. Technological choices: transformation in material cultures since the Neolithic. Routledge, London, UK. https://doi. org/10.4324/9781315887630

van der Leeuw, S. E. 2007. Information processing and its role in the rise of the European world system. Pages 213-241 in R. Costanza, L. J. Graumlich, W. Steffen, editors. Sustainability or collapse? MIT Press, Cambridge, Massachusetts, USA.

van der Leeuw, S. E. 2012. For every solution there are many problems: the role and study of technical systems in socioenvironmental coevolution. Danish Journal of Geography 112 (2):105-116. https://doi.org/10.1080/00167223.2012.741887

van der Leeuw, S. E. 2020a. The role of narratives in humanenvironmental relations: an essay on elaborating win-win solutions to climate change and sustainability. Climatic Change 160:509-519. https://doi.org/10.1007/s10584-019-02403-y

van der Leeuw, S. E. 2020b. Social sustainability, past and future: undoing unintended consequences for the Earth's survival. Cambridge University Press, Cambridge, UK. https://doi. org/10.1017/9781108595247

Vitali, S., J. B. Glattfelder, and S. Battiston. 2011. The network of global corporate control. PLoS ONE 6(10):e25995. https://doi. org/10.1371/journal.pone.0025995

Westley, F., K. McGowan, and O. Tjornbo, editors. 2017. The evolution of social innovation. Edward Elgar, Cheltenham, UK. https://doi.org/10.4337/9781786431158

Westley, F. R., O. Tjörnbo, L. Schultz, P. Olsson, C. Folke, B. Crona, and Ö. Bodin. 2013. A theory of transformative agency in linked social-ecological systems. Ecology and Society 18(3):27. https://doi.org/10.5751/ES-05072-180327

Westley, F., P. Olsson, C. Folke, T. Homer-Dixon, H. Vredenburg, D. Loorbach, J. Thompson, M. Nilsson, E. Lambin, J. Sendzimir, B. Banarjee, V. Galaz, and S. van der Leeuw. 2011. Tipping toward sustainability: emerging pathways of transformation. Ambio 40:762. https://doi.org/10.1007/s13280-011-0186-9

Wyer, R. S. 2009. Culture and information processing: a conceptual integration. Pages 431-456 in R. S. Wyer, C. Chiu, and Y. Hong, editors. Understanding culture: theory, research and application. Psychology Press, New York, New York, USA. 


\section{Appendix 1. Glossary.}

Attractor: In the mathematical field of dynamical systems, an attractor is a set of numerical values toward which a system tends to evolve, for a wide variety of starting conditions of the system. System values that get close enough to the attractor values remain close even if slightly disturbed. In non-mathematical terms, as used here, an attractor is a (set of) state(s) of a dynamic physical system toward which that system tends to evolve, regardless of the starting conditions of the system.

Basin of attraction: It is very common for dynamical systems to have more than one attractor. For each such attractor, its basin of attraction is the set of initial conditions leading to long-time behavior that approaches that attractor. Thus the qualitative behavior of the long-time motion of a given system can be fundamentally different depending on which basin of attraction the initial condition lies in (e.g., attractors can correspond to periodic, quasiperiodic or chaotic behaviors of different types). Regarding a basin of attraction as a region in the state space, it has been found that the basic topological structure of such regions can vary greatly from system to system.

Bifurcation points: Points in a system's trajectory where attractors and repellors collide or cancel each other out, or where different attractors pull in different directions, causing a loss of stability so that it is not evident where the system's dynamic will move next. One could think of a bifurcation point as a 'decision moment' for a system. The simplest example is a fold bifurcation involving a saddle point and a node. This is where the classical basin of attraction shrinks to nil and disappears. However, one can also have an unstable and a stable cycle collide, or have strange attractors collide with a range of unstable structures.

Cognitive space, and its spheres: The virtual space in which information-processing takes place in society. We propose the following structure of that cognitive space into three spheres. The core is composed of stable, well-balanced and developed mental formations in which closed categories dominate. The developing component around the core is composed of mental formations at the stage of coming into being, dominated by open categories. Around that sphere, we assume a range finds a sphere where there are no mental formations yet coming into being, but there is awareness of phenomena dimly perceived. This is the sphere of the known unknown. The information processing in the cognitive space is fundamental to the ways in which societies interact with their basins of attraction.

Entropy: There are two equivalent definitions of entropy: the thermodynamic definition and the statistical mechanics definition. In the classical thermodynamics viewpoint, the microscopic details of a system are not considered. Instead, the behavior of a system is described in terms of a set of empirically defined thermodynamic variables, such as temperature, pressure, entropy, and heat capacity. The interpretation of entropy in statistical mechanics is the measure of uncertainty, diffuseness or mixedupness, which remains about a system after its observable macroscopic properties, such as temperature, pressure and volume, have been taken into account. For a given set of macroscopic variables, the entropy measures the degree to which the probability of the system is spread out over different possible microstates. In this paper we look at the difference between the expected (macrostate) entropy of the way in which an interviewee describes certain phenomena, (which can be calculated on the basis of known and observable average variables), and the observed (microstate) entropy, which measures the actual description of the phenomena concerned. That difference is here considered as a proxy measure of the degree of uncertainty the interviewee has about the phenomena concerned. 
Niche construction: In biology, the process by which an organism alters its own local environment (Odling Smee et al. 2003). These alterations can be a physical change to the organism's environment or encompass what occurs when an organism actively moves from one habitat to another to experience a different environment. Examples of niche construction include the building of nests and burrows by animals, and the creation of shade, influencing of wind speed, and alternation of nutrient cycling by plants. Although these alterations are often beneficial to the constructor they are not always (for example, when organisms dump detritus they can degrade their own environments). In this paper, the processing of information is considered to be a case of niche construction in which the human (or societal) information processing apparatus shapes and is shaped by the environment (in the wide sense) in which the society is embedded.

Nonlinearity: Describes a situation where there is not a straight-line or direct relationship between an independent variable and a dependent variable. In a nonlinear relationship, the output does not change in direct proportion to a change in any of the inputs. It may either change faster, so-called super-linearly, or slower, sub-linearly. Both cases frequently occur in complex systems. For example, when looking at the dynamics of urban systems, the surface of cities changes, according to any appropriate proxy measure, linearly with the size of the population, but energy use, as measured by electricity use, changes sub-linearly and information-processing changes super-linearly. The interaction between super-linear, linear and sub-linear dynamics can drive the system in very complex ways.

Resonance: Technically describes the phenomenon of increased amplitude that occurs when the frequency of a periodically applied force (or a Fourier component of it) is equal or close to a natural frequency of the system on which it acts. Thus, when an oscillating force is applied at a resonant frequency of a dynamical system it is some sense 'in tune' with the system. The system will therefore oscillate at a higher amplitude than when the same force is applied at other, non-resonant frequencies. In this paper we use resonance to indicate interactions in which the observation of outside phenomena reinforces some aspects of a society's cognitive structure, while other aspects of the observation do not and they challenge that structure. Such challenges will trigger changes in the cognitive structure that enrich it.

Technosphere: The domain that encompasses all of the technological objects manufactured by humans, but that is only part of it. It is a system, and not just a growing collection of technological hardware. It includes the goals, functions, dynamics and other aspects of the functioning of human systems in so far as they are determined by technology. Forest ecosystems, animals and machines, nanotechnology, the internet, highways, medical systems, power grids, human populations, political parties, governments and bureaucracies, robots and religions and their interactions with each other all impact on the dynamics of societal systems. In the technosphere approach, humans and their ideas are therefore part and parcel of the technosphere. Its dynamic is neither uniquely material nor uniquely societal, but truly technical - in the sense that existing technology is to an important extent considered to determine human thinking and behavior as well as the future development of technology.

Tipping point: A critical threshold in a system's dynamic at which a tiny perturbation can qualitatively or structurally alter the state or structure of a system. The term is particularly used with respect to climate change, indicating points on the temperature scale where the impact of climate change is expected to result in dramatic changes in many aspects of the living systems that sustain human existence on Earth. In this paper, the term indicates points in a system's trajectory where its information processing has to be dramatically restructured, not unlike a 'paradigm 
change' in the sense of Kuhn (1962). It is argued that this is often the case due to an accumulation of unexpected consequences of earlier system dynamics leading to what is experienced as a crisis, an incapacity of an existing information processing system to deal with the information needed to allow the system to have a harmonious interaction with its environment. 